\title{
ÉTAT DE LITTÉRATURE. DÉCONSTRUIRE POUR DÉNONCER : LA TRAITE DES ÊTRES HUMAINS EN DÉBAT
}

\section{Milena Jakšić}

\author{
Presses de Sciences Po | « Critique internationale »
}

2011/4 n 53 | pages 169 à 182

ISSN 1290-7839

ISBN 9782724632231

Article disponible en ligne à l'adresse :

https://www.cairn.info/revue-critique-internationale-2011-4-page-169.htm

Distribution électronique Cairn.info pour Presses de Sciences Po.

(C) Presses de Sciences Po. Tous droits réservés pour tous pays.

La reproduction ou représentation de cet article, notamment par photocopie, n'est autorisée que dans les limites des conditions générales d'utilisation du site ou, le cas échéant, des conditions générales de la licence souscrite par votre établissement. Toute autre reproduction ou représentation, en tout ou partie, sous quelque forme et de quelque manière que ce soit, est interdite sauf accord préalable et écrit de l'éditeur, en dehors des cas prévus par la législation en vigueur en France. Il est précisé que son stockage dans une base de données est également interdit. 


\section{État de littérature \\ Déconstruire pour dénoncer : la traite des êtres humains en débat}

\section{par Milena Jakšić}

la traite des êtres humains. Selon sa définition juridique, l'expression désigne le commerce des personnes en vue notamment de l'exploitation sexuelle, du travail forcé, de l'esclavage et du prélèvement d'organes ${ }^{1}$. C'est pourtant sur la seule traite à des fins d'exploitation sexuelle que l'attention se concentre pour dénoncer ce qui est décrit comme l'une des pires atteintes aux droits de l'homme : être acheté, être vendu, être exploité.

Les chercheurs en sciences sociales n'échappent pas à cet attrait pour un sujet qui depuis la fin du XIXe siècle ${ }^{2}$ 《sollicite tous les refoulements ${ }^{3}$. L'historien Alain Corbin a d'ailleurs été l'un des premiers à souligner le « pouvoir émotionnel» de la traite qui fait que «les esprits les plus pondérés s'égarent $\gg^{4}$. Les chercheurs s'égarent, eux aussi, et peinent à se démarquer de la grammaire dans laquelle la traite et ses victimes sont énoncées au sein d'un forum hybride où experts, lobbies, médias et décideurs politiques débattent et s'affrontent pour instituer le phénomène en «cause qui parle ${ }^{5}$. Cette

\footnotetext{
1. Pour une analyse de l'évolution des normes législatives en matière de traite au niveau européen et international, voir Anne T. Gallagher, The International Law of Human Trafficking, Cambridge, Cambridge University Press, 2010 ; Birgit Locher, Trafficking in Women in the European Union: Norms, Advocacy-Networks and Policy-Change, Wiesbaden, VS Verlag, 2007. Pour une analyse de la législation française en matière de traite, avec le nombre d'affaires portées en justice, voir Johanne Vernier, La traite et l'exploitation des êtres humains en France, Paris, La Documentation française, 2010. Pour une mise en perspective historique, voir le travail du sociologue Jean-Michel Chaumont qui a livré une analyse des travaux du Comité spécial d'experts (CSE) chargé par la Société des Nations (SDN) de superviser une enquête internationale sur la traite des femmes et des enfants, de 1924 à 1927. Jean-Michel Chaumont, Le mythe de la traite des blanches. Enquête sur la fabrication d'un fléau, Paris, La Découverte, 2009.

2. D'après l'historien Edward W. Bristow, le terme de « traite des blanches » apparaît pour la première fois en 1839 , dans un contexte marqué par l'antisémitisme. Les juifs et les étrangers sont alors désignés comme les principaux porteurs de ce nouveau «fléau ». Edward W. Bristow, Prostitution and Prejudice: Fewish Fight Against White Slavery, 1870-1939, Oxford, Clarendon Press, 1982.

3. Alain Corbin, Les filles de noce : misère sexuelle et prostitution, XIX et XX $X^{e}$ siècles, Paris, Flammarion, 1982, p. 426.

4. A. Corbin souligne par ailleurs que la traite mobilise, dès son apparition, les plus grandes peurs, en faisant jouer « la xénophobie, l'antisémitisme, l'anticléricalisme ou bien encore l'anxiété diffuse concernant la dépopulation du pays et l'abâtardissement de la race ». Ibid., p. 405.

5. Annie Collovald, Brigitte Gaïti, « Des causes qui "parlent”... », Politix, 4 (16), 1991, p. 7-22.
} 
difficulté à se distancier du point de vue normatif et expert est particulièrement prégnante dans la majorité des travaux qui sont parus depuis la fin des années 1990. Dans ce premier corpus d'analyses qui oscillent entre dénonciation indignée, dévoilement critique et expertise militante, tout se passe comme si le chercheur devait nécessairement prendre position, diagnostiquer le problème et en proposer une solution. Cela est d'autant plus vrai que la traite à des fins d'exploitation sexuelle est prise dans de véritables sex wars ${ }^{6}$ où s'affrontent deux courants aux positions inconciliables : celui des féministes abolitionnistes, qui appréhendent la prostitution et la traite en termes de violences à l'encontre des femmes, et celui des féministes d'inspiration libérale, qui revendiquent la liberté de disposer de son corps ${ }^{7}$.

La construction de l'objet d'étude diffère sensiblement en fonction de la ligne idéologique adoptée. Les travaux qui s'inspirent de l'approche abolitionniste mettent l'accent sur les caractéristiques sociographiques de la victime, décrite comme une jeune femme naïve et vulnérable qui doit être sauvée. Les inégalités Nord/Sud, la légalisation de la prostitution et le statut subordonné des femmes sont tenus pour les causes structurelles d'un phénomène dénoncé comme la face la plus sordide de «l'esclavage moderne $»^{8}$. À l'inverse, les travaux se plaçant dans une perspective qui pourrait être qualifiée de « prodroits $\gg^{9}$ mettent l'accent sur les notions d'agency et d'empowerment, en référence à la capacité des acteurs sociaux d'influer sur les rapports de pouvoir dans lesquels ils sont pris. Dans cette seconde perspective, la catégorie de victime, jugée misérabiliste et victimisante, est déconstruite et remplacée par celle des prostituées migrantes à qui les États dénient le droit d'émigrer ou d'exercer librement ce que certaines d'entre elles considèrent comme un métier légitime. Pour les tenants de cette approche, les politiques restrictives

\footnotetext{
6. Le sociologue Ronald Weitzer déconstruit et dénonce l'effet « contaminant» des sex wars sur l'objet traite. Voir notamment Ronald Weitzer, «The Growing Moral Panic over Prostitution and Sex Trafficking», The Criminologist, 30 (5), septembre-octobre 2005, p. 2-5 ; « The Social Construction of Sex Trafficking: Ideology and Institutionalization of a Moral Crusade », Politics \& Society, 35, 2007, p. 447-475. De la même manière, pour Lilian Mathieu, les études sur la prostitution oscillent bien souvent entre «misérabilisme » et «populisme ». Lilian Mathieu, La condition prostituée, Paris, Textuel, 2007.

7. Cette opposition est qualifiée de «Yalta féministe » par Éric Fassin dans « Le genre aux États-Unis », dans Christine Bard, Christian Baudelot, Janine Mossuz-Lavau (dir.), Quand les femmes s'en mêlent. Genre et pouvoir, Paris, Éditions de La Martinière, 2004, p. 23-44.

8. Pour les écrits les plus emblématiques de cette approche, voir Donna M. Hughes, « The "Natasha" Trade: The Transnational Shadow Market of Trafficking in Women », Journal of International Affairs, 53 (2), été 2000, p. 625651 ; Sheila Jeffreys, The Industrial Vagina: The Political Economy of the Global Sex Trade, New York, Routledge, 2009 ; Richard Poulin (dir.), La mondialisation des industries du sexe, Paris, Imago, 2005.

9. Marieke van Doorninck, Marjan Wijers, « Only Rights Can Stop Wrongs: A Critical Assessment of Anti-Trafficking Strategies », EU/IOM STOP European Conference on Preventing and Combating Trafficking in Human Beings - A Global Challenge for the $21^{\text {st }}$ Century, Bruxelles, 18-20 septembre 2002.
} 
à l'égard de l'immigration et le contrôle de l'activité prostitutionnelle sont responsables de la condition subordonnée des femmes ${ }^{10}$.

Cependant, quels que soient leurs partis pris idéologiques, les défenseurs de ces deux courants ont en commun de privilégier les thèses militantes et partisanes au détriment de la démonstration empirique des faits. L'une des explications potentielles de cet égarement des chercheurs est que beaucoup d'entre eux sont impliqués dans des réseaux de militantisme ou dans des coalitions d'advocacy qui participent à la constitution même du phénomène qu'ils se donnent pour objet d'analyse. Il n'empêche qu'en privilégiant des points de vue partisans, ces travaux, qui dominent très largement le champ des études sur la traite, participent à la problématisation des enjeux et à la constitution des réseaux d'expertise. Leur restitution critique dans le cadre de l'état de littérature présenté ici en dit autant sur la manière dont la traite a été constituée en cause que sur la grammaire dans laquelle le phénomène est énoncé par les chercheurs en sciences sociales. Elle est donc indispensable pour mettre en lumière les écueils potentiels qui guettent une question placée au cœur de l'actualité.

Le deuxième corpus étudié ici se détache en partie - et en partie seulement de cette posture normative. Mobilisant politistes et sociologues, ces travaux offrent des analyses riches et pertinentes en termes de régimes d'énonciation de la traite, de labellisation des enjeux et de constitution des réseaux d'expertise. L'une des limites de cette perspective tient cependant au fait qu'elle s'appuie essentiellement sur l'analyse des discours sans parvenir à explorer de manière plus systématique et rigoureuse les répertoires d'action mobilisés ou les propriétés sociographiques des acteurs engagés. L'impact des programmes anti-traite en termes de modalités de prise en charge des personnes est un autre angle mort de ce deuxième corpus d'analyses. Il convient donc de croiser et de compléter celui-ci par une troisième série de travaux, plus rares, qui privilégient une approche ethnographique par le bas, à partir d'entretiens avec des personnes désignées communément comme « victimes de la traite ». Cependant, malgré la richesse du matériau recueilli, ces travaux se réclamant d'une approche délibérément «déconstructiviste» n'échappent pas à quelques-unes des apories qui entravent les analyses des chercheurs pris dans les sex wars des débats sur la traite.

10. Ce point de vue est défendu par Laura Agustìn, Sex at the Margins. Migration, Labour, Markets and the Rescue Industry, Londres, Zed Books, 2007. 


\section{Entre expertise indignée et expertise critique : les travaux pionniers sur la traite}

Nous l'avons dit, l'approche normative des abolitionnistes comme des prodroits domine très largement la littérature contemporaine sur la traite. Leurs positions alimentent en effet les controverses qui animent l'espace des mobilisations depuis le début des années $1980^{11}$.

La première approche, celle des abolitionnistes, devient manifeste dès 1984, date à laquelle la revue Nouvelles Questions féministes ${ }^{12}$ consacre un numéro spécial aux actes du colloque dit de Rotterdam qui, à l'instigation de Kathleen Barry ${ }^{13}$, lance le Réseau féministe international contre l'esclavage sexuel et la traite des femmes. En 1988, ce dernier devient la Coalition Against Trafficking in Women (CATW), principale organisation abolitionniste dans la lutte contre la traite des femmes. La position défendue par les chercheuses abolitionnistes transparaît dès l'introduction à ce numéro, sous la plume des sociologues Christine Delphy et Claude Faugeron. En associant la traite à la prostitution et à la violence à l'encontre des femmes, C. Delphy et C. Faugeron revendiquent le droit de parler à la place des opprimées, afin de dénoncer ce qui est désigné comme une «oppression des femmes ». Cette dénonciation experte au nom des opprimées devient la ligne directrice de la littérature abolitionniste. La thèse selon laquelle les hommes utilisent le sexe pour affirmer leur domination sur les femmes s'impose comme le cadre théorique pour penser les phénomènes de traite et de prostitution. Ce cadre comprend quatre grands points : 1) la prostitution est une «institution de la domination sexuelle» et une «autre forme de viol ${ }^{14}$. Il s'ensuit que la traite à des fins d'exploitation sexuelle est inséparable de la question prostitutionnelle; 2) la violence, l'absence de choix et l'emploi de la contrainte sont intrinsèques à la traite et à la prostitution ; 3) la pauvreté et les politiques légalisant la prostitution sont les principaux facteurs responsables de cette oppression ; 4) les personnes prostituées sont les victimes d'un système dans lequel elles sont entraînées contre leur volonté (puisque la prostitution ne saurait relever d'un choix).

11. Pour une analyse de ces controverses, voir Louise Toupin, La question du « trafic des femmes ». Points de repères dans la documentation des coalitions féministes internationales anti-trafic, Montréal, Stella, 2002.

12. Kathleen Barry, Charlotte Bunch, Shirley Castley, Matsui Yayori, Hanna Olsson (dir.), « Féminisme international : réseau contre l'esclavage sexuel : rapport de l'atelier féministe international contre la traite des femmes. Rotterdam/Pays-Bas 6-15 avril 1983 », numéro spécial de Nouvelles Questions féministes, 8, 1984.

13. Sociologue et figure emblématique du féminisme abolitionniste, auteur notamment de L'esclavage sexuel de la femme, Paris, Stock, 1982 (1 1 ère édition 1979).

14. K. Barry, « La prostitution est un crime », Déviance et société, 10 (3), 1986, p. 301-302. 
Ce cadre théorique a des implications importantes en termes de conduite des enquêtes de terrain. L'expertise indignée transparaît avec emphase et véhémence sous la plume du sociologue Richard Poulin qui fait un usage immodéré de la surgénéralisation pour dénoncer le pire des «fléaux »: «le système prostitutionnel ${ }^{15}$. Cette littérature se distingue par un recours abusif à l'outil statistique visant à démontrer la validité des thèses abolitionnistes. Nous apprenons ainsi que « $90 \%$ des personnes victimes de la traite le sont à des fins de prostitution $»$ (p. 9), qu'entre « $80 \%$ et $90 \%$ des personnes prostituées en Occident ont été agressées sexuellement dans leur jeunesse » (p. 16) et qu'entre « $85 \%$ et $90 \%$ des personnes prostituées sont sous la coupe d'un proxénète ou d'un réseau de proxénètes $\gg$ (p. 18). Et R. Poulin de s'interroger : « Dans de telles conditions, peut-on soutenir qu'il y a vraiment une prostitution "libre", volontairement choisie ? » (p. 17). Sa réponse et celle d'autres chercheurs d'orientation abolitionniste est très clairement négative.

Parallèlement à cette volonté de formuler des diagnostics sociaux, le trait distinctif de cette littérature prolifique est de passer systématiquement sous silence les conditions de production de ces données chiffrées, en ignorant - délibérément ? les travaux qui mettent en cause leur validité ${ }^{16}$. Or nous savons bien, depuis au moins les travaux d'Alain Desrosières ${ }^{17}$, à quel point la statistique, cette mise en chiffres de la société, loin de refléter le « problème social » contribue au contraire à le définir. Dans la littérature abolitionniste sur la traite, l'usage des données statistiques fait de la violence et de l'emploi de la contrainte, non pas des hypothèses de recherche à mettre à l'épreuve des enquêtes de terrain, mais des réalités avérées qu'il appartient au sociologue de révéler.

En réponse à cette approche jugée misérabiliste et victimisante, une abondante littérature s'attache depuis la fin des années 1990 à distinguer «la nature d'une activité » de ses conditions d'exercice ${ }^{18}$. Ses auteurs, membres ou proches de la Global Alliance Against Trafficking in Women (GAATW) et du Network for Sex Work Projects (NSWP), les deux organisations rivales de la CATW, estiment que seul l'usage de la contrainte est à retenir comme

15. R. Poulin, Prostitution, la mondialisation incarnée : points de vue du Sud, Louvain-la-Neuve/Paris, Centre tricontinental/Syllepse, 2005.

16. Liz Kelly, « "You Can Find Anything You Want": A Critical Reflection on Research on Trafficking in Persons within and into Europe », International Migration, 43 (1/2), 2005, p. 235-265 ; Guri Tyldum, Anette Brunovskis, «Describing the Unobserved: Methodological Challenges in Empirical Studies on Human Trafficking », International Migration, 43 (1/2), 2005, p. 17-34.

17. Alain Desrosières, La politique des grands nombres, Paris, La Découverte, 1993.

18. Kamala Kempadoo, Jo Doezema (eds), Global Sex Workers: Rights, Resistance, and Redefinition, New York, Londres, Routledge, 1998 ; Kamala Kempadoo, Jyoti Saghera, Bandana Pattanaik (eds), Trafficking and Prostitution Reconsidered: New Perspectives on Migration, Sex Work, and Human Rights, Londres, Paradigm Publishers, 2005. 
critère de définition des situations de traite. Ce sont des juristes, des sociologues ou des chercheuses engagées dans des départements d'études féministes et de théories du genre qui, toutes, ont une double casquette de chercheuse et de militante/experte. Leurs thèses peuvent également être résumées en quatre grands points : 1) distinguer la traite de la prostitution, soit les situations où la contrainte est impliquée du choix d'exercer librement l'activité prostitutionnelle ; 2) autonomiser la traite par rapport à la question prostitutionnelle pour $\mathrm{y}$ inclure d'autres formes de travail forcé; 3) démontrer que l'argument de lutte contre la traite sert en réalité de justification aux politiques restrictives à l'égard de l'immigration et de la prostitution ${ }^{19}$; 4) déconstruire la catégorie homogénéisante et restrictive des « victimes de la traite » pour faire émerger des histoires singulières qui mettent en lumière le rôle actif des femmes dans le processus migratoire. Ainsi, plutôt que de dénoncer les violences endurées, il s'agit pour ces auteurs de mettre l'accent sur l'importance d'octroyer des droits aux travailleurs migrants et aux personnes prostituées.

Or cette littérature pro-droits pose, elle aussi, d'importants problèmes méthodologiques, dans la mesure où elle s'appuie sur la seule analyse des discours ou des programmes anti-traite, avec la volonté marquée de discréditer les positions de la partie adverse ${ }^{20}$. Comment dans ce contexte des sex wars proposer une lecture qui se démarquerait de ce parti pris idéologique?

\section{Sortir des sex wars : une analyse des régimes d'énonciation de la traite}

Ce défi est relevé par le deuxième ensemble de travaux qui prend pour objet d'analyse les régimes d'énonciation de la traite et la constitution des réseaux d'expertise. Ces chercheurs, issus du champ de la science politique et de la sociologie, tentent de reconstituer le répertoire sémantique sur lequel s'appuient les acteurs soit pour distinguer, soit pour revendiquer une situation de traite. Cette approche les engage à poser une série de questions : quelles

\footnotetext{
19. Sur ce point de vue, voir notamment les travaux de J. Doezema, présidente du NSWP, qui met également en garde contre les effets pervers de la distinction prostitution libre/forcée. Pour elle, cette distinction risque d'introduire la séparation entre «prostituées coupables» et «victimes innocentes ». J. Doezema, « Forced to Choose: Beyond the Voluntary v. Forced Prostitution Dichotomy», dans K. Kempadoo, J. Doezema (eds), Global Sex Workers: Rights, Resistance, and Redefinition, op. cit., p. 46-47.

20. Sur cette posture, voir Melissa Ditmore, Marjan Wijers, « The Negotiations on the UN Protocol on Trafficking in Persons. Moving the Focus from Morality to Actual Conditions », Nemesis, 4, 2003, p. 79-88. Voir également J. Doezema, «Ouch! Western Feminists "Wounded Attachment" to the "Third World Prostitute" », Feminist Review, 67, 2001, p. 16-38; « Now You See Her, Now You Don't: Sex Workers at the UN Trafficking Protocol Negotiations », Social \& Legal Studies, 14 (1), 2005, p. 61-89, et notamment son dernier ouvrage Sex Slaves and Discourse Masters: The Construction of Trafficking, Londres, Zed Books, 2010.
} 
sont les possibilités et les difficultés d'usage du terme lui-même ? Quels sont les lieux d'usage du terme, avec ses acceptations et ses résistances? Quelles sont les potentialités du phénomène et qui sont les principaux promoteurs de la cause ? Les terrains permettant d'éprouver cette série de questions sont multiples. On retrouve d'abord des travaux qui prennent pour objet d'analyse les débats parlementaires ou les politiques législatives en matière de traite et de prostitution. Que ce soit en Grande-Bretagne ${ }^{21}$, aux Pays-Bas ${ }^{22}$, aux ÉtatsUnis $^{23}$ ou en France ${ }^{24}$, ces travaux montrent, dans leur ensemble, que les arguments anti-traite sont systématiquement mobilisés en vue de distinguer les prostituées «nationales » des prostituées étrangères, de criminaliser la prostitution de rue ou encore de justifier les retours dits volontaires des prostituées étrangères sous prétexte qu'elles seraient victimes des réseaux de criminalité organisée. Ces écrits sont donc unanimes pour dire que la lutte contre «l'esclavage sexuel » est devenue simultanément un instrument légitime non seulement de normalisation de la sexualité, mais aussi de contrôle de l'immigration et de la libre circulation des personnes.

D'autres travaux s'intéressent à la mise en place des programmes anti-traite au sein de l'Union européenne et sur la scène des mobilisations internationales ${ }^{25}$. La politiste Nadège Ragaru propose ainsi une analyse très fine des « récits de déclinaison de la traite $\gg$ depuis le début des années $1990{ }^{26}$. À partir d'une étude des «querelles interprétatives» qui opposent les principaux acteurs enrôlés dans la cause de la traite des êtres humains, elle montre que l'émergence du «problème traite » ne suit pas une logique linéaire «dans laquelle plusieurs étapes obligées auraient été franchies - l'émergence des pratiques de traite, la prise de conscience internationale grâce aux efforts de sensibilisation d'acteurs sociaux variés, la formulation de réponses au "fléau" progressivement

\footnotetext{
21. Johanna Kantola, Judith Squires, « Discourses Surrounding Prostitution Policies in the UK», European Fournal of Women's Studies, 11 (1), 2004, p. 77-101.

22. Joyce Outshoorn, « Debating Prostitution in Parliament: A Feminist Analysis », European Fournal of Women's Studies, 8 (4), 2001, p. 472-490; « The Political Debates on Prostitution and Trafficking in Women », Social Politics: International Studies in Gender, State and Society, 12 (1), 2005, p. 141-155.

23. Wendy Chapkis, « Trafficking, Migration and the Law: Protecting Innocents, Punishing Immigrants », Gender and Society, 17 (6), 2003, p. 923-937; Gretchen Soderlund, « Running from the Rescuers: New U.S. Crusades against Sex Trafficking and the Rhetoric of Abolition », NWSA fournal, 17 (3), p. 64-87 ; Yvonne C. Zimmermann, «From Bush to Obama. Rethinking Sex and Religion in the United States' Initiative to Combat Human Trafficking », fournal of Feminist Studies in Religion, 26 (1), 2010, p. 79-99.

24. Milena Jakšić, « Figures de la victime de la traite des êtres humains : de la victime idéale à la victime coupable », Cabiers internationaux de sociologie, 124, 2008, p. 127-146.

25. Pour une analyse des programmes anti-traite en Europe et en Asie du Sud-Est, voir Leslie Holmes (ed.), Trafficking and Human Rights: European and Asia-Pacific Perspectives, Cheltenham, Edward Elgar, 2010.

26. Nadège Ragaru, « Du bon usage de la traite des êtres humains. Controverses autour d'un problème social et d'une qualification juridique », Genèses, 66, 2007, p. 69-89; « La traite des êtres humains : histoire d'une mise sur agenda international », Regard sur l'Est, 2004 (http://www.regard-est.com/home/breve_contenu.php?id=494).
} 
affinées ${ }^{27}$. Il semble au contraire que nous soyons en présence « de reformulations constantes d'un objet aux contours mouvants, à la faveur de l'entrée en scène d'un nombre toujours plus grand d'acteurs », de sorte que la cause traite ressemble davantage à un «marché - très compétitif - de savoirs et savoirfaire, de positions normatives et de financements $\gg^{28}$. Son analyse de l'activité des principales organisations intergouvernementales, gouvernementales et non gouvernementales met en évidence un décalage important entre les logiques internes de ces organisations, leurs règles de conduite et la réalité du terrain. Elle observe l'« autonomisation grandissante [des programmes anti-traite] par rapport au terrain et, surtout, par rapport aux vécus des pratiques de traite par les personnes qui, qualifiées de "victimes", se voient proposer diverses prises en charge ${ }^{29}$. Enfin, elle souligne l'importance d'entreprendre des études empiriques sur « les conditions de construction de la figure de la "victime" (...) et [sur] la manière dont elles déshumanisent les destinataires de l'assistance $\gg^{30}$. L'effet déshumanisant des programmes anti-traite trouve en partie son explication dans la tension inhérente aux deux discours qui dominent les politiques anti-traite : le discours humanitaire, dans lequel la traite est avant tout appréhendée comme une menace pour le corps vulnérable des femmes, et le discours sécuritaire, qui, en associant la traite à la prostitution et à l'immigration clandestine, appréhende le problème en termes de menace pour la souveraineté des États. Ainsi, les politiques de lutte contre la traite associent dans le même mouvement un discours humanitaire de défense des droits de l'homme et un discours sécuritaire de défense de l'ordre public et des priorités nationales. C'est du moins la thèse défendue par la politiste Claudia Aradau ${ }^{31}$, qui place le discours humanitaire du côté des politiques de la pitié ${ }^{32}$ et le discours sécuritaire du côté des politiques du risque, en insistant sur leurs liens d'interdépendance ${ }^{33}$. Cette thèse est également défendue par la politiste Mathilde Darley, qui souligne comment « dans un contexte politique voué tout entier à la lutte contre la "menace" et à "l'éradication du risque”, la traite n'est plus seulement perçue par les États comme une atteinte à l'individu, appelant une réponse humanitaire, mais aussi comme une des

27. N. Ragaru, « Du bon usage de la traite des êtres humains. Controverses autour d'un problème social et d'une qualification juridique », art. cité, p. 70 .

28. Ibid., p. 71.

29. Ibid., p. 85.

30. Ibid..

31. Claudia Aradau, Retbinking Trafficking in Women. Politics out of Security, New York, Palgrave Macmillan, 2008.

32. En mobilisant notamment les travaux de Luc Boltanski sur la morale humanitaire. Luc Boltanski, La souffrance à distance. Morale humanitaire, médias et politique, Paris, Métailié, 1993.

33. C. Aradau, «The Perverse Politics of Four-Letter Words: Risk and Pity in the Securisation of Human Trafficking », Millenium: Fournal of International Studies, 33 (2), 2004, p. 251-277. 
formes d'expression du crime organisé et de l'immigration clandestine, et donc comme une atteinte à l'intégrité du territoire ${ }^{34}$. Ce point de vue est partagé par la politiste Jacqueline Berman, qui, à l'appui des travaux de Michel Foucault ou d'Anne Stoler, tente de montrer comment les mesures anti-traite contribuent à former un nouveau régime biopolitique ${ }^{35}$, fondé sur une «politique de l'altérité » qui établit une distinction entre les femmes occidentales, seules dotées de la «puissance d'agir», et les « autres », contenues dans la catégorie homogénéisante des victimes de la traite et présentées comme des sujets passifs et vulnérables qui méritent protection et justifient l'intervention de l'État sur leurs vies ${ }^{36}$.

Si ces travaux apportent des éclairages intéressants pour comprendre la problématisation des enjeux qui entourent la question de la traite, certaines de leurs limites méritent d'être soulignées. La principale est sans doute la personnification de l'Europe et des institutions qui la composent. «L'Europe fait », «Europol décide » sont des formules récurrentes. À l'inverse, et à l'exception du travail de N. Ragaru, les caractéristiques sociographiques des acteurs mobilisés, les contraintes auxquelles ils sont soumis et le dispositif de prise de décision sont insuffisamment explorés. Cette personnification des institutions est corrélative de la tendance à leur attribuer une intentionnalité et une rationalité propres au nom d'un objectif clairement établi, mais qui reste caché (ici, la criminalisation des prostituées et des migrants serait l'objectif implicite des politiques anti-traite). Or personnifier les institutions et homogénéiser l'ensemble des acteurs qui composent l'espace européen et/ ou international dans la lutte contre la traite revient à faire l'économie d'une analyse des incertitudes, des hésitations et des tensions qui animent ces acteurs dont les responsabilités, les objectifs et les statuts sont inégaux. Cela revient également à faire l'impasse sur l'analyse de la carrière des institutions engagées dans la lutte contre la traite, carrière qui retrace une histoire de la mobilisation qu'il serait intéressant de restituer. Enfin, ces approches qui se fondent sur la seule analyse des discours ou des campagnes anti-traite ne s'interrogent pas suffisamment sur la mise en œuvre effective des programmes véhiculés par ces discours.

\footnotetext{
34. Mathilde Darley, « Le statut de la victime dans la lutte contre la traite des femmes », Critique internationale, 30, janvier-mars 2006, p. 108.

35. Jacqueline Berman, «Biopolitical Management, Economic Calculation and "Trafficked Women" », International Migration, 48, 2010, p. 84-113.

36. J. Berman, «(Un)Popular Strangers and Crises (Un)Bounded: Discourses of Sex-Trafficking, the European Political Community and the Panicked State of the Modern State », European fournal of International Relations, 9 (1), 2003, p. 37-86.
} 


\section{Des enquêtes ethnographiques par le bas}

Cette limite est en partie comblée par la troisième série de travaux « déconstructivistes» qui, au-delà de la simple analyse des discours, se fondent sur des enquêtes ethnographiques, croisant observation participante et entretiens semi-directifs avec les femmes concernées par les programmes anti-traite. La thèse de Rutvica Andrijašević constitue à cet égard l'exemple le plus édifiant ${ }^{37}$. Entre octobre 1999 et novembre 2000, elle a conduit une enquête à Bologne, en Italie, auprès de 25 femmes migrantes, originaires de l'Europe de l'Est, des pays non-candidats à l'UE et qui, au moment des entretiens, avaient toutes cessé de travailler comme prostituées sur la voie publique. L'objectif de R. Andrijašević est d'opposer les récits de ces femmes aux discours et aux représentations relatifs à la traite des femmes, tels qu'ils apparaissent dans la presse et dans les rapports d'expertise produits par les plus grandes instances intergouvernementales et non gouvernementales. Son enquête ethnographique apporte de précieux éléments qui contredisent l'image dominante des victimes de la traite en tant que sujets passifs et ignorants. Elle explique ainsi que «contrairement à l'idée selon laquelle les femmes sont toujours contraintes à une immigration illégale par les trafiquants, quelques-unes racontent comment elles ne parvinrent à réaliser leur projet de départ qu'avec l'aide de passeurs $\gg^{38}$. Le passeur apparaît avant tout comme un pourvoyeur de ressources, comme l'ultime voie de secours dans un contexte où il est devenu impossible ou du moins particulièrement difficile de franchir les frontières légalement. R. Andrijašević montre néanmoins que le lien de cause à effet entre l'immigration illégale et la traite n'est pas toujours fondé : de nombreuses femmes entrent en Italie légalement, munies d'un visa souvent touristique, mais se retrouvent dans la condition de sans-papiers après que celui-ci a dépassé sa durée de validité.

R. Andrijašević déconstruit l'image de la femme «dupée » et «trompée » souvent attribuée à la victime de la traite ${ }^{39}$. Son étude montre que de nombreuses femmes avaient entrepris volontairement le projet migratoire en vue

37. Rutvica Andrijašević, Migration, Agency and Citizenship in Sex Trafficking, New York, Palgrave Macmillan, 2010. 38. R. Andrijašević, « La traite des femmes d'Europe de l'Est en Italie. Analyse critique des représentations », Revue européenne des migrations internationales, 21 (1), 2005, p. 160.

39. Pour une analyse des campagnes anti-traite, productrices de cette image dominante, voir Céline Nieuwenhuys, Antoine Pécoud, « Human Trafficking, Information Campaigns, and Strategies of Migration Control », American Behavioral Scientist, 50 (12), 2007, p. 1674-1695; «Campagnes d'information et traite des êtres humains à l'Est de l'Europe », Espace, populations, sociétés, 2, 2008, p. 319-330. 
de travailler dans l'industrie du sexe ${ }^{40}$ « mais qu'elles n'étaient pas toujours au courant des conditions de vie et de travail qui les attendaient dans le pays de destination $\gg^{41}$. Selon elle, le jugement moral qui entoure la prostitution et la condamnation de la violence qu'elle génère contribuent à enfermer les prostituées migrantes dans la catégorie stigmatisante de victimes ignorantes et occultent dans le même mouvement le vécu de ces femmes et leur capacité à objectiver leur propre expérience. Les entretiens conduits auprès des femmes migrantes montrent au contraire comment «l'entrée en Italie par le biais de la traite était [pour elles] un moyen d'être mobile et d'émigrer $\gg^{42}$. Parmi les travaux menés en France, citons l'enquête ethnographique de Françoise Guillemaut qui, tout en formulant des thèses similaires à celles de R. Andrijašević, se place dans un registre volontairement militant. En mobilisant les apports de la sociologie du genre et des études féministes ou des black feminists, elle cherche à déconstruire la catégorie jugée dépolitisante des «victimes de la traite» pour lui opposer la figure de migrantes autonomes pour qui la prostitution constitue avant tout un choix et une ressource économique $^{43}$. Afin de repérer les décalages entre «l'expérience des femmes » et « les discours sur la prostitution et le trafic », elle fonde donc son analyse sur vingt récits de vie de « femmes primo-migrantes autonomes, prostituées, exprostituées et non prostituées ${ }^{44}$. Elle offre ainsi un tableau vivant des migrations « pour soi » et fait apparaître les migrantes comme des sujets agissants, capables de choisir et de développer des «stratégies de vie» de manière indépendante ${ }^{45}$. Les notions d'agency et d'empowerment, centrales dans cette lecture, sont mobilisées dans le but de déconstruire le regard «misérabiliste» et «sexiste» des politiques anti-traite. F. Guillemaut

\footnotetext{
40. D'autres travaux soutiennent l'idée de la prostitution comme ressource migratoire. Voir Florence Lévy, Marylène Lieber, « La sexualité comme ressource migratoire. Les Chinoises du Nord de Paris », Revue française de sociologie, 50 (4), 2009, p. 719-746. Pour une analyse associant les notions de contrainte et de ressource, voir Vanessa Simoni, «Territoires et enjeux de pouvoir de la traite aux fins d'exploitation sexuelle : le cas de Paris », Hérodote, 136, 2010, p. 134-149.

41. R. Andrijašević, «La traite des femmes d'Europe de l'Est en Italie. Analyse critique des représentations», art. cité, p. 162.

42. Ibid., p. 163.

43. Françoise Guillemaut, «Victimes de trafic ou actrices d'un processus migratoire ? Saisir la voix des femmes migrantes prostituées par la recherche-action », Terrains \& Travaux, 1 (10), 2006, p. 157-176 ; « Sexe, juju et migrations. Regard anthropologique sur les processus migratoires de femmes africaines en France », Recherches sociologiques et anthropologiques, 1, 2008, p. 10-25.

44. F. Guillemaut, «Stratégies des femmes en migration : pratiques et pensées minoritaires. Repenser les marges au centre », thèse de doctorat en sociologie et sciences sociales, université Toulouse II, 2007.

45. On trouve d'autres exemples des migrations « pour soi » dans Laura Oso Casa, « Migration et trafic des femmes latino-américaines en Espagne : service domestique et prostitution », Les Cabiers du CEDREF, 2003, p. 163-186, et dans Nasima Moujoud, Dolorès Pourette, « "Traite" des femmes migrantes, domesticité et prostitution », Cabiers d'études africaines, XLV (3-4), 2005, p. 1093-1221.
} 
démontre que « cet écart entre les discours et la réalité révèle une constante qui est celle de l'invisibilisation des migrantes autonomes comme de l'agentivité des femmes $\gg^{46}$. Les programmes anti-traite obéissent en réalité à la logique du «not in my backyard » pour la prostitution et du « keep your women at home » pour les migrations ${ }^{47}$. Et c'est la propulsion de la figure de la victime qui autorise, selon l'auteur, le contrôle de la sexualité des femmes et de leurs mouvements.

\section{Des pistes de recherche}

Dans l'ensemble, ces travaux s'attachent à repérer des écarts et des décalages entre la constitution de la traite en cause internationale et le vécu des personnes auxquelles sont destinés les programmes anti-traite. Leur contribution la plus importante est de déconstruire la catégorie homogénéisante et universaliste des « victimes de la traite », pour lui opposer des récits de vie de femmes migrantes qui révèlent une réalité plus complexe, faite de choix, de projets autonomes et de désirs migratoires qui peuvent inclure le travail dans l'industrie du sexe.

Tout en reconnaissant l'apport de ces recherches à la sociologie de la traite des êtres humains, il convient de souligner certaines de leurs limites. Ces travaux, qui se placent dans une perspective déconstructiviste, cherchent en réalité à comprendre comment la traite et ses victimes sont socialement construites. Ils proposent une explication en termes de mobilisations sociales, de législations, de travail de lobbying, d'expertise et d'activité de militants, d'avocats et d'organismes internationaux. Or ce matériau à la fois riche et fécond amène in fine certains de ces auteurs à conclure que ce qui est socialement construit ne correspond pas à la réalité. Le «construit» devient ici synonyme de «faux ${ }^{48}$. Et pour dévoiler cette «fausseté » afin de montrer où se situe la réalité des choses, ils mobilisent des récits de femmes migrantes censés incarner le vrai. Cette démarche correspond à ce que Ian Hacking appelle le «constructivisme de dévoilement» qu'il définit comme une volonté de «démasquer une idée non pas tant pour la "désintégrer" mais pour la dépouiller de ses faux attraits ou de toute autorité » ${ }^{49}$. Privée de ses faux-semblants et de ses parasites, la traite apparaît alors comme un simple

46. F. Guillemaut, « Stratégies des femmes en migration : pratiques et pensées minoritaires. Repenser les marges au centre $\gg$, cité, p. 558.

47. F. Guillemaut, « Trafics et migrations de femmes, une hypocrisie au service des pays riches », Hommes et migrations, 1248, mars-avril 2004, p. 75-89.

48. Outre par J.-M. Chaumont, la traite est également qualifiée de « mythe » par J. Doezema dans « Loose Women or Lost Women? The Re-emergence of the Myth of "White Slavery" in Contemporary Discourses of "Trafficking in Women" », Gender Issues, 18 (1), 2000, p. 23-50.

49. Ian Hacking, Entre science et réalité. La construction sociale de quoi ? Paris, La Découverte, 2001, p. 37. 
instrument au service de causes «cachées», comme le contrôle de l'immigration ou la pénalisation des personnes prostituées. La catégorie de victime, quant à elle, est systématiquement mise entre guillemets ou tout simplement remplacée par d'autres syntagmes, pour se démarquer de la dépolitisation que le terme engendre. Mais la finalité de la démarche sociologique vise-t-elle bien à dévoiler le « faux » ? Et, d'abord, faut-il considérer le construit comme nécessairement faussé ou artificiel ?

Afin d'éviter ces écueils, les enquêtes à venir pourraient s'articuler autour de trois principaux axes : la production des catégories (de victime, de clandestin, de travailleur exploité) ; la traduction de ces catégories dans des structures et des institutions ; la manière dont les individus sont affectés par ces catégories. Cependant, loin de postuler une logique séquentielle entre ces trois niveaux d'analyse, des allers et retours, des croisements et des interactions entre la production des catégories et leur institutionnalisation méritent d'être questionnés. Car les catégories et les classifications qui les produisent n'existent jamais dans un «empty space of language », pour reprendre une formule de I. Hacking, elles ont besoin d'institutions, de pratiques, d'interactions matérielles avec des objets et des personnes.

Ainsi, si les travaux présentés ici ont contribué à déconstruire les discours sur la traite, discours entendus au sens foucaldien de «pratiques qui forment systématiquement les objets dont ils parlent $\gg^{50}$, il nous faut désormais orienter nos recherches vers des analyses qui s'attacheraient à reconstituer la carrière des personnes prises en charge par les programmes anti-traite, sans succomber à la tentation d'invalider ou de dénoncer a priori la qualité ou l'efficacité de ces programmes. Il s'agirait plutôt de multiplier les enquêtes ethnographiques en étudiant le type de relations nouées entre les institutions et les personnes désignées comme victimes. Cette approche pourrait apporter des réponses riches et constructives pour la compréhension du «travail juridico-bureaucratique de catégorisation $\gg^{51}$ de personnes dépourvues des ressources nécessaires à la défense de leur cause et qui sont, de plus, catégorisées à la fois comme victimes d'une des pires atteintes aux droits de l'homme et comme coupables de séjour irrégulier et de racolage. Dans quelles conditions les victimes de la traite peuventelles accéder concrètement au statut d'ayant droit? Quelles contraintes de justification et quels modèles de compétences ces opérations de tri et de cadrage des individus supposent-elles ? Comment un jugement sur les personnes est-il arrêté

50. Michel Foucault, L'archéologie du savoir, Paris, Gallimard, 1969, p. 66-67.

51. Gérard Noiriel, « Représentation nationale et catégories sociales. L'exemple des réfugiés politiques », Genèses, 26 (1), 1997, p. 27. 
et comment est-il remis en cause ? Telles sont quelques-unes des questions dont l'exploration pourrait se révéler féconde.

Milena Jakšić est docteur en sociologie de l'École des hautes études en sciences sociales. En croisant enquête ethnographique et sociologie des mobilisations et de l'action publique, elle s'est intéressée à la construction de la catégorie de la victimecoupable de la traite. Elle a notamment publié « Figures de la victime de la traite des êtres humains : de la victime idéale à la victime coupable ", Cahiers internationaux de sociologie (124, 2008, p. 127-146).

Adresse électronique : milenajaksic@gmail.com 